\title{
Solutions for nonlinear convection in the presence of a lateral boundary
}

\author{
P.G. Daniels, D. Ho \& A.C. Skeldon ${ }^{1,2}$ \\ Department of Mathematics, City University, Northampton Square, London EC1V
}

$O H B$

\begin{abstract}
A method is described for calculating steady-state patterns in large-scale nonlinear systems, taking into account the effect of a lateral boundary and without the need for extensive numerical calculations. The key feature is the determination of the phase shift of the nonlinear periodic form distant from the boundary as a function of wavelength. This is found by analyzing the solution close to the boundary, where Floquet theory is used to describe the departure of the solution from its periodic form. For a restricted band of wavelengths lying within the Eckhaus boundary, dual solutions for the phase shift are found, one of which corresponds to an unstable state. Results are presented here for the one-dimensional Swift-Hohenberg equation in a semi-infinite domain but in principle the method can be applied to more general pattern-forming systems. The results are compared with the predictions of weakly nonlinear theory and with nonlinear computations on a large but finite domain.
\end{abstract}

Key words: convection, influence of boundaries, Swift-Hohenberg equation

\section{Introduction}

There are many examples of systems that develop a cellular pattern through a bifurcation or smooth evolution from a structureless state. Such patterns have been observed in the hydrodynamic instability of simple fluids (such as

1 Present address: Department of Mathematics and Statistics, University of Surrey, Guildford, Surrey GU2 7XH, UK.

2 Corresponding author. Tel. +44 1483 682634; fax. +44 1483 876071; email: a.skeldon@surrey.ac.uk

Preprint submitted to Elsevier Science 13 December 2007 
Rayleigh-Bénard convection and Taylor-Couette flow), in the electrohydrodynamic instability of liquid crystals, in crystal growth and in the buckling of plates (see, for example, [1]). A key issue in predicting pattern formation in such systems is to understand how the presence of lateral boundaries affects the structure, orientation and wavelength of the pattern. It is known from experimental work on Rayleigh-Bénard and other systems that such boundaries generally have a strong influence, even in large-aspect-ratio systems [2], but numerical investigations are hampered by the immense computing power needed to accurately simulate three-dimensional flows in the fully nonlinear regime (see, for example, [3]). Thus a number of phenomenological models have been studied as a means of gaining insight into the nonlinear regime. The following model equation was first introduced by Swift and Hohenberg $[4]$ :

$$
\frac{\partial u}{\partial t}=\varepsilon u-\left(1+\nabla^{2}\right)^{2} u-u^{3}
$$

Here $t$ is time, $\varepsilon$ is a control parameter and $u=u(x, y, t)$ is a two-dimensional scalar field which is intended to represent a characteristic property of the convection pattern, such as the vertical velocity component at mid-height in a horizontal layer parallel to the $x, y$ plane. The Swift-Hohenberg equation has been much studied in the last twenty years. Cross et al $[5,6]$ investigated the effect of lateral boundaries on solutions of the one-dimensional equation in the weakly nonlinear regime $(\varepsilon \ll 1)$. They showed that even if the lateral walls are far (many roll-widths) apart, they severely restrict the band of allowed wavenumbers in the bulk of the fluid compared with that which exists for the corresponding infinite layer. Kramer and Hohenberg [7] used a numerical method to find results for two isolated values of $\varepsilon$ in the nonlinear regime and also determined the Eckhaus stability boundary for $0<\varepsilon \leq 1$. Further details of the bifurcation structure and Eckhaus instability mechanism were discussed by Tsiveriotis and Brown [8], whilst Hernandez-Garcia et al [9] considered the influence of noise on pattern selection. A comprehensive numerical study of the two-dimensional Swift-Hohenberg equation was reported by Greenside et al [10] and Greenside and Coughran [11], and Kuwamura [12] considered the stability of roll solutions of the two-dimensional equation using a spectral analysis. 
In the present paper the weakly nonlinear results of Cross et al [6] for the onedimensional Swift-Hohenberg equation are extended to the nonlinear regime using a method based on Floquet analysis. Steady-state solutions are sought in a semi-infinite domain $x \geq 0$ which adjust to a lateral boundary at $x=0$ and approach a periodic form as $x \rightarrow \infty$, equivalent to a roll pattern parallel to the boundary in the corresponding Rayleigh-Bénard problem. The aim is to develop a method that allows the classification of such solutions in the nonlinear regime in terms of their phase shift relative to the boundary. Once this phase shift is known, the nonlinear solution is effectively determined far from the boundary by its periodic extension, avoiding the need for a numerical representation other than within a few roll-widths of the boundary itself. An extension of this approach to the Rayleigh-Bénard system would offer the prospect of avoiding extensive three-dimensional numerical calculations in large-aspect-ratio systems.

The first step is to determine the steady-state nonlinear spatially-periodic solutions of the one-dimensional Swift-Hohenberg equation. Some of these have been discussed by Kramer and Hohenberg [7] although even in the onedimensional case there is a myriad of bifurcation structures that result from mode interactions and lead to a non-uniqueness of periodic states at given values of $\varepsilon$ and the wavenumber $q$. Dangelmayr [13] and Cox [14] have considered similar mode interactions in Rayleigh-Bénard convection. Results for the onedimensional Swift-Hohenberg equation are determined here using a shooting method and are described in Section 2. In a semi-infinite domain $0 \leq x<\infty$ the presence of a lateral boundary at $x=0$ introduces a spatial perturbation to the periodic form that can be analyzed using Floquet theory. This analysis is described in Section 3 and leads to the determination of a set of Floquet exponents and eigenfunctions for each nonlinear solution at given points in the $\varepsilon, q$ plane. A bi-product of this analysis is the location of the Eckhaus stability boundary at which the spatial perturbation becomes oscillatory, but within the Eckhaus boundary the existence of a spatially-decaying mode signals the possibility of a stable steady-state solution that satisfies the relevant conditions at the lateral boundary $x=0$. In effect, this provides the asymptotic form of the solution as $x \rightarrow \infty$ in which the amplitude of the perturbation and the phase shift of the periodic form must then be chosen to ensure that the boundary conditions are satisfied at $x=0$. This procedure is carried out 
in Section 4 and leads to the classification of dual solutions within a restricted region of the $\varepsilon, q$ plane. At each stage, the results are carefully compared with those of weakly nonlinear analysis in the limit $\varepsilon \rightarrow 0$, and stability arguments are used to show that one of the dual solutions is unstable. The results are discussed in Section 5.

\section{Periodic solutions}

In this section steady-state periodic solutions of the one-dimensional SwiftHohenberg equation

$$
\frac{\partial u}{\partial t}=(\varepsilon-1) u-\frac{\partial^{4} u}{\partial x^{4}}-2 \frac{\partial^{2} u}{\partial x^{2}}-u^{3}
$$

are found. It is convenient to introduce a positive parameter $L$ and use the boundary conditions

$$
u=\frac{\partial^{2} u}{\partial x^{2}}=0 \quad \text { at } \quad x=0, L
$$

in which case the wavenumber of the solution is given by $q=n \pi / L$ for some positive integer $n$.

\subsection{Linear analysis}

Infinitesimal perturbations from the trivial solution $u=0$ are governed by the linearized version of the system (2), (3) which has normal-mode solutions

$$
u=\mathrm{e}^{s t} \sin \frac{n \pi x}{L}, \quad n=1,2, \ldots
$$

with growth rates $s=\varepsilon-\left((n \pi / L)^{2}-1\right)^{2}$. Thus the trivial solution becomes unstable when

$$
\varepsilon=\left(\left(\frac{n \pi}{L}\right)^{2}-1\right)^{2} .
$$




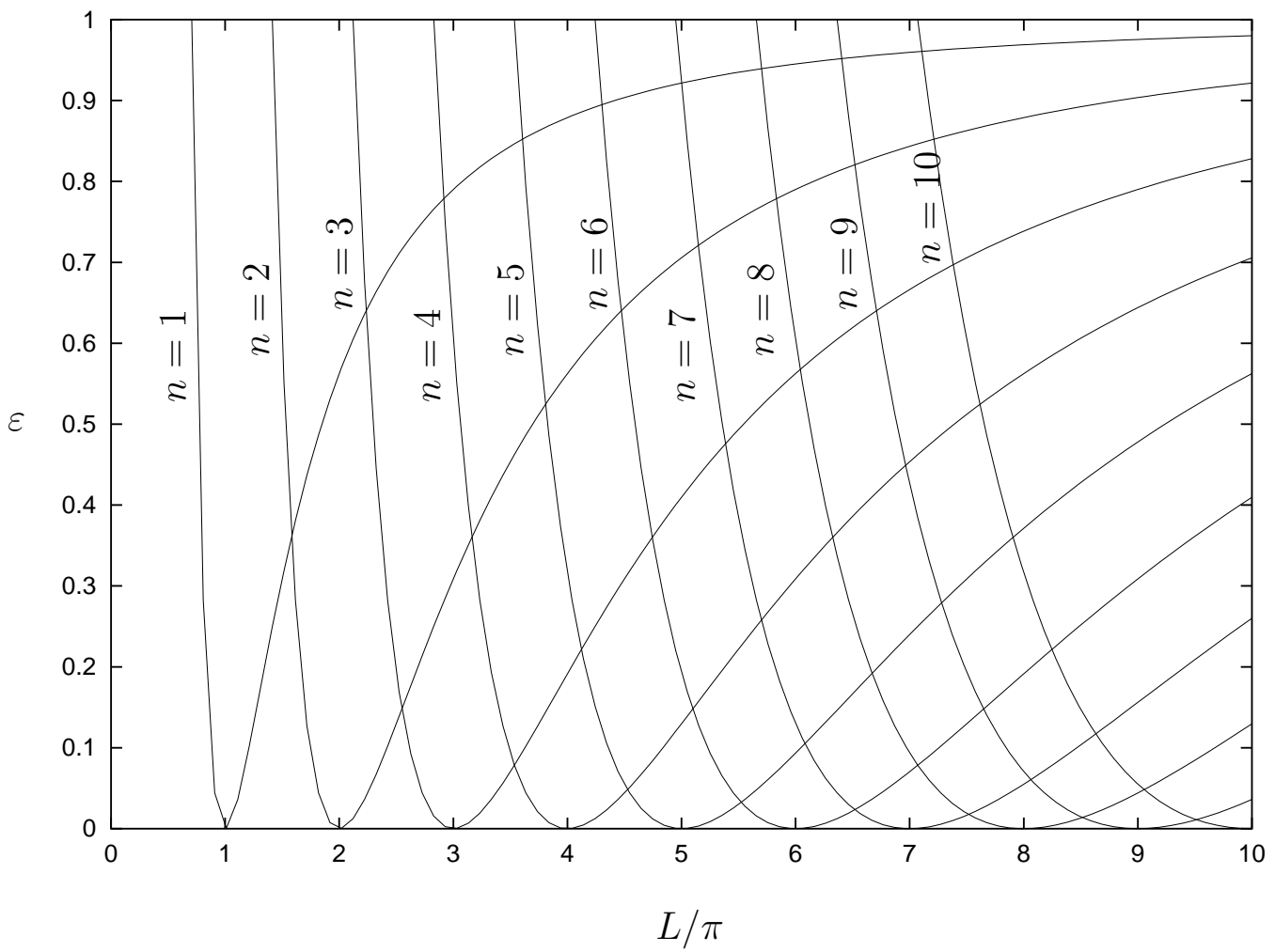

Fig. 1. Marginal curves for $n=1,2 \ldots 10$.

Figure 1 shows these marginal curves, which identify the onset of nonlinear periodic solutions, for the first ten modes $n=1,2, \ldots 10$. Although these curves each represent the onset of the same pair of periodic solutions (proportional to $\sin \sqrt{\left(1 \pm \varepsilon^{1 / 2}\right)} x$ ) at a given value of $\varepsilon$, their intersection points herald the development of new nonlinear solutions through mode interactions which are discussed in more detail below.

\subsection{Weakly nonlinear analysis $\varepsilon \ll 1$}

The linear analysis shows that as $\varepsilon$ increases from zero, steady-state periodic solutions with small amplitude first exist in the neighbourhood of the critical point with wavenumber $q=1$. Locally the solution for $u$ can be developed in the form

$$
u=\varepsilon^{1 / 2} u_{0}+\varepsilon u_{1}+\varepsilon^{3 / 2} u_{2}+\varepsilon^{2} u_{3}+\ldots, \quad \varepsilon \rightarrow 0,
$$


where $u_{i}=u_{i}(x, X, \tau)$ are functions of $x$ and the long length and time scales $X=\varepsilon^{1 / 2} x$ and $\tau=\varepsilon t$. Substitution into (2) then yields in succession

$$
\begin{aligned}
\frac{\partial^{4} u_{0}}{\partial x^{4}}+2 \frac{\partial^{2} u_{0}}{\partial x^{2}}+u_{0} & =0 \\
\frac{\partial^{4} u_{1}}{\partial x^{4}}+2 \frac{\partial^{2} u_{1}}{\partial x^{2}}+u_{1} & =-4\left(\frac{\partial^{4} u_{0}}{\partial x^{3} \partial X}+\frac{\partial^{2} u_{0}}{\partial x \partial X}\right) \\
\frac{\partial^{4} u_{2}}{\partial x^{4}}+2 \frac{\partial^{2} u_{2}}{\partial x^{2}}+u_{2}= & -4\left(\frac{\partial^{4} u_{1}}{\partial x^{3} \partial X}+\frac{\partial^{2} u_{1}}{\partial x \partial X}\right)-6 \frac{\partial^{4} u_{0}}{\partial x^{2} \partial X^{2}} \\
& -\frac{\partial u_{0}}{\partial \tau}-2 \frac{\partial^{2} u_{0}}{\partial X^{2}}+u_{0}-u_{0}^{3} .
\end{aligned}
$$

Solving equation (7), the relevant periodic form is

$$
u_{0}=A_{0}(X, \tau) \mathrm{e}^{i x}+c . c .
$$

where $A_{0}$ is a complex amplitude function and c.c. denotes complex conjugate. It now follows from (8) that

$$
u_{1}=A_{1}(X, \tau) \mathrm{e}^{i x}+\text { c.c. }
$$

where $A_{1}$ is a further complex amplitude function, and then in order that $u_{2}$ is periodic the secular terms proportional to $\mathrm{e}^{ \pm i x}$ on the right-hand side of (9) must vanish, in which case $A_{0}$ satisfies

$$
\frac{\partial A_{0}}{\partial \tau}=4 \frac{\partial^{2} A_{0}}{\partial X^{2}}+A_{0}-3 A_{0}\left|A_{0}\right|^{2}
$$

Steady-state periodic solutions for $u$ are now found by assuming that

$$
A_{0}=A_{p}(X)=R \mathrm{e}^{i Q X}
$$

where $R$ is constant. The conditions (3) then imply that $R$ is purely imaginary and that $Q$ must satisfy

$$
L\left(1+Q \varepsilon^{1 / 2}\right)=n \pi, \quad n=1,2 \ldots
$$

Finding $R= \pm i\left(1-4 Q^{2}\right)^{1 / 2} / \sqrt{3}$ from (12), it follows that (to within an arbitrary change of sign) weakly nonlinear steady-state periodic solutions have 
the form

$$
u=u_{p}(x) \sim 2 \sqrt{\frac{\varepsilon}{3}}\left(1-4 Q^{2}\right)^{1 / 2} \sin \frac{n \pi x}{L}, \quad-\frac{1}{2} \leq Q \leq \frac{1}{2}
$$

as $\varepsilon \rightarrow 0$, where $Q$ and $L$ are related by (14). These solutions exist inside the marginal stability curves, which correspond to the limiting values $Q= \pm \frac{1}{2}$.

\subsection{Nonlinear solutions}

A shooting method was used to obtain steady periodic solutions $u=u_{p}(x)$ of the full nonlinear problem (2), (3). The steady-state version of equation (2) was converted into a set of four first order ordinary differential equations and solutions obtained by a fourth order Runge-Kutta scheme starting from conditions

$$
\left(u, u_{x}, u_{x x}, u_{x x x}\right)=(0, \alpha, 0, \beta) \quad \text { at } x=0 .
$$

The values of $u$ and its first three derivatives are monitored at $x=L$ and a Newton iteration used to adjust the values of $\alpha$ and $\beta$ until $u$ and $u_{x x}$ vanish there. Solutions were found in the $\varepsilon, L$ plane starting at $\varepsilon=0.1, \quad L=\pi$ where initial estimates of $\alpha$ and $\beta$ are provided by the weakly nonlinear solution (15), (14). The ODE solver within Matlab's ODESUITE [15] was used and further solutions were then obtained by incrementing $L$ or $\varepsilon$, using results from the previous calculation as initial estimates for $\alpha$ and $\beta$. The pathfollowing code used a heuristic method to increment the step size in $L$ or $\varepsilon$ : the number of Newton iterates is monitored and the step size reduced or increased accordingly.

Figure 2 shows plots of $\alpha$ and $\beta$ for $2<L<7.5$ and values of $\varepsilon$ in the range 0.01 to 0.65 . Profiles of $u$ at $L=\pi$ over a much wider range of $\varepsilon$ are shown in Figure 3. For low values of $\varepsilon$ there is a single nonlinear solution (apart form an arbitrary change of sign) at each point in the $\varepsilon, L$ plane, but at $\varepsilon=9 / 25$ further solutions are generated by the 1-2 mode interaction at $L=\sqrt{10} \pi / 2$. The resulting nonlinear solutions bifurcate from the basic nonlinear solution in the manner seen in Figure 2 for $\varepsilon=0.4,0.6$ and 0.65 . 


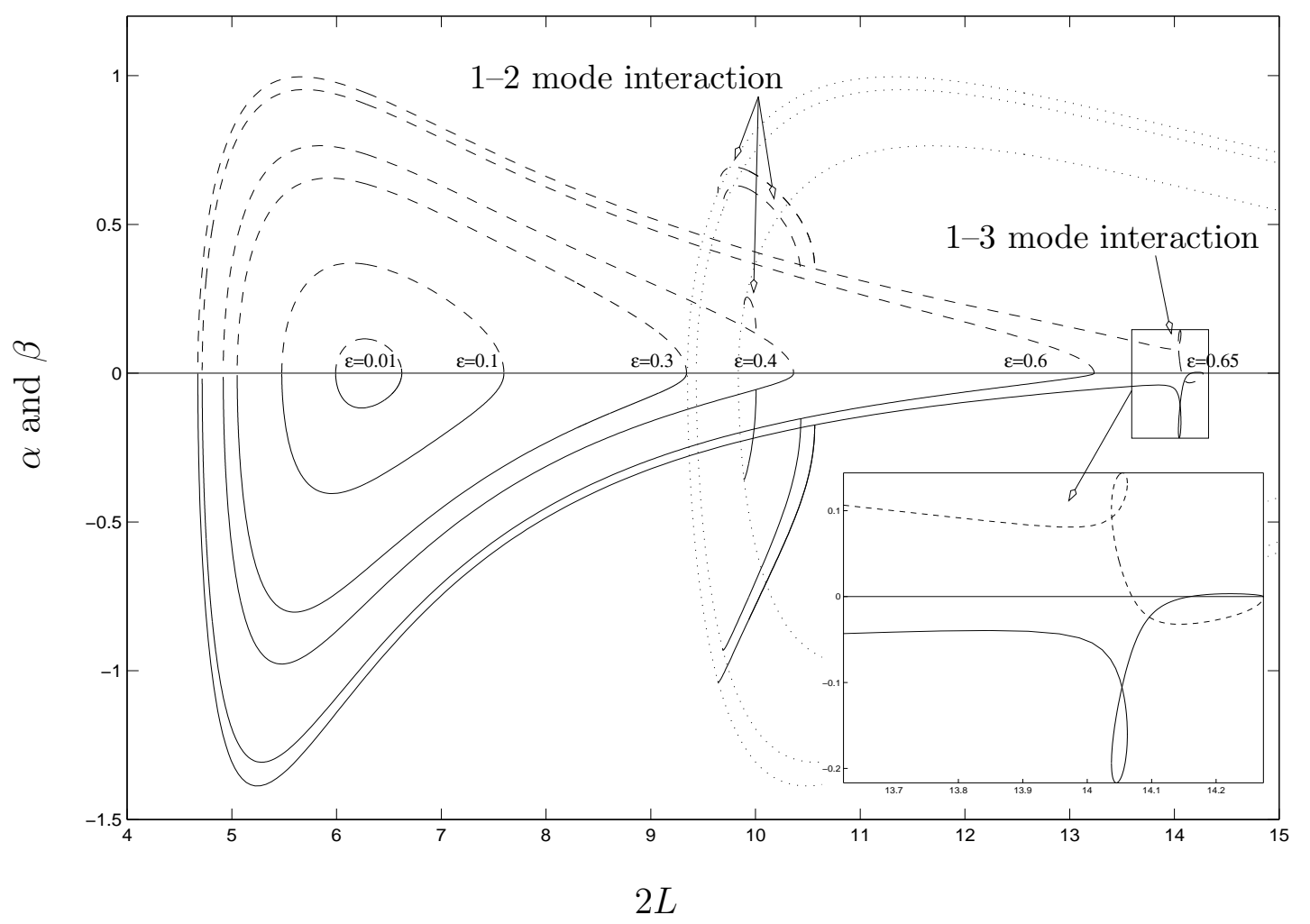

Fig. 2. Parameters $\alpha\left(-_{-}\right)$and $\beta(-)$ as a function of $L$ for $\varepsilon=0.01,0.1,0.3,0.4,0.6$ and 0.65 . The dotted lines $(\cdots)$ correspond to the $n=2$ mode.

The next mode interaction is the 1-3 interaction which is initiated at $\varepsilon=16 / 25$, $L=\sqrt{5} \pi$. The intricate structure of the resulting nonlinear solution is seen in Figure 2 for $\varepsilon=0.65$ and leads to the existence of three nonlinear solutions within a restricted range of $L$. Here the cubic nonlinearity of the SwiftHohenberg equation leads to a resonance in which the mode 3 component evolves smoothly with increasing $L$, rather than through a bifurcation from the 1-mode solution. This can be seen in the local profiles of $u$ shown in Figure 4.

Local analyses of the 1-2 and 1-3 mode interactions were carried out to confirm and assist in the numerical computations and are described in detail by Ho [16]. 

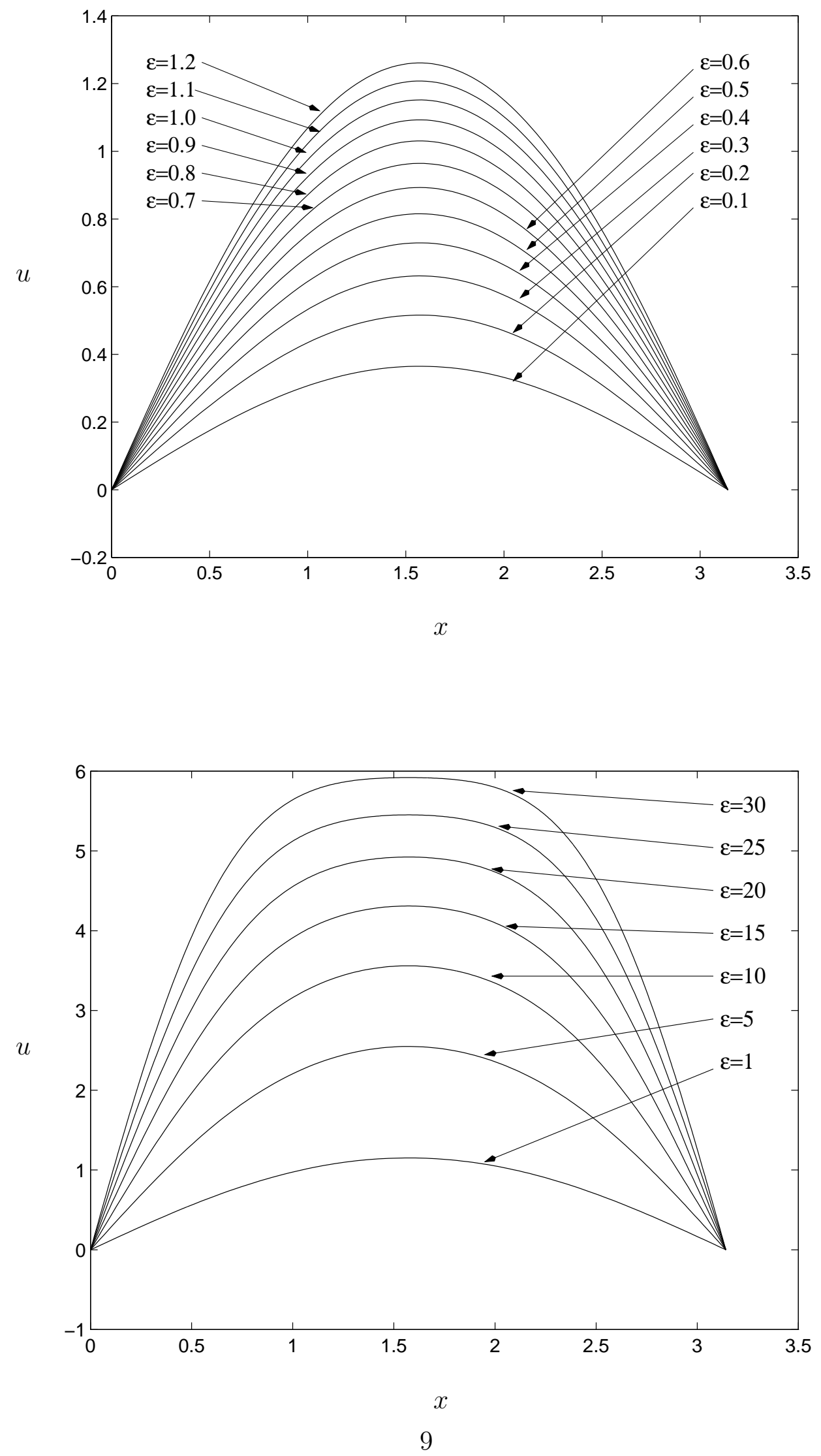

Fig. 3. Profiles of the periodic solution $u(x)$ for $L=\pi$ and $\varepsilon$ in the range 0.1 to 30 . 


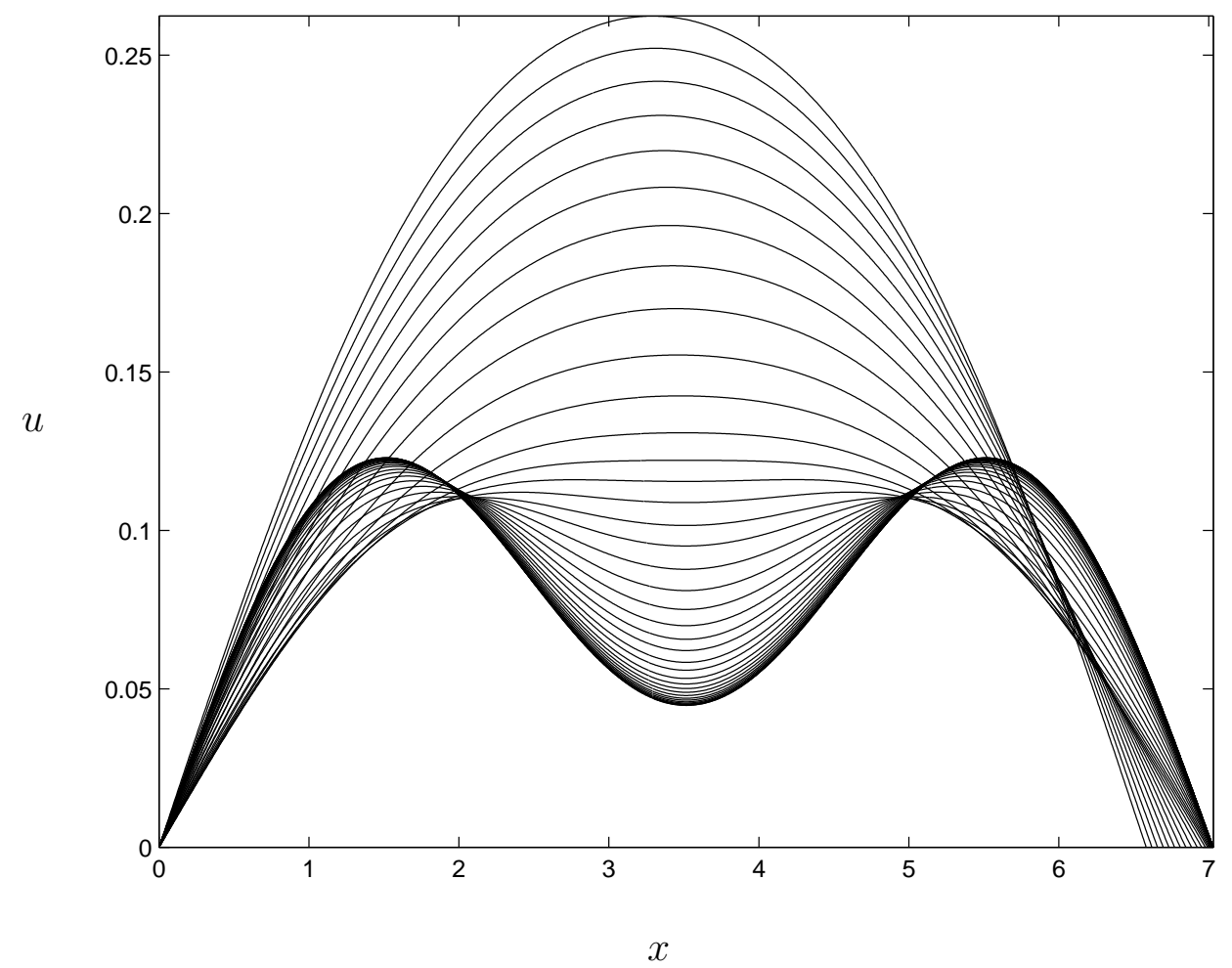

Fig. 4. Profiles of the periodic solution $u(x)$ for $\varepsilon=0.65$ and values of $L$ near 7 .

\section{Floquet analysis}

Steady-state perturbations to the periodic solutions determined in the previous section are now considered by writing

$$
u=u_{p}(x)+\tilde{k} U(x)+\ldots,
$$

where the constant $\widetilde{k} \ll 1$ and $u_{p}$ denotes the periodic solution. Substitution into (2) and neglecting nonlinear terms in $\widetilde{k}$ shows that the function $U$ satisfies

$$
\left(\frac{d^{2}}{d x^{2}}+1\right)^{2} U-\varepsilon U+3 u_{p}^{2} U=0
$$

Since (18) is a linear ordinary differential equation with periodic coefficients it follows from Floquet theory (see, for example, [17]) that there are solutions 
of the form

$$
U(x)=\mathrm{e}^{-c x} P(x)
$$

where $P(x)$ is periodic with the same period as that of $u_{p}$. Note that if the characteristic exponent $c$ or eigenfunction $P$ is complex then the conjugate of $\widetilde{k} U$ is also a solution and can be added in (17) to ensure that $u$ is real. In order to find $c$ and $P$, the solution for $U$ is written in the form

$$
U=\sum_{i=1}^{4} \alpha_{i} U_{i}(x),
$$

where $U_{i}$ are four linearly independent solutions of (18) obtained by, in turn, using the initial conditions $U_{1}(0)=1, \quad U_{2}^{\prime}(0)=1, \quad U_{3}^{\prime \prime}(0)=1$ and $U_{4}^{\prime \prime \prime}(0)=1$. In each case, all other values of $U_{i}$ and its first three derivatives are taken to be zero at $x=0$. Use of (19) together with the periodicity conditions

$$
\frac{d^{j} P}{d x^{j}}(0)=\frac{d^{j} P}{d x^{j}}(2 L), \quad j=0,1,2,3
$$

then gives

$$
\left(\begin{array}{llll}
\left(U_{1}-\mathrm{e}^{-2 c L}\right) & U_{2} & U_{3} & U_{4} \\
U_{1}^{\prime} & \left(U_{2}^{\prime}-\mathrm{e}^{-2 c L}\right) & U_{3}^{\prime} & U_{4}^{\prime} \\
U_{1}^{\prime \prime} & U_{2}^{\prime \prime} & \left(U_{3}^{\prime \prime}-\mathrm{e}^{-2 c L}\right) & U_{4}^{\prime \prime} \\
U_{1}^{\prime \prime \prime} & U_{2}^{\prime \prime \prime} & U_{3}^{\prime \prime \prime} & \left(U_{4}^{\prime \prime \prime}-\mathrm{e}^{-2 c L}\right)
\end{array}\right)\left(\begin{array}{l}
\alpha_{1} \\
\alpha_{2} \\
\alpha_{3} \\
\alpha_{4}
\end{array}\right)=\left(\begin{array}{l}
0 \\
0 \\
0 \\
0
\end{array}\right)
$$

where $U_{i}$ and its derivatives are evaluated at $x=2 L$. Non-trivial solutions correspond to zeros of the determinant of the coefficient matrix, and this leads to the evaluation of the Floquet exponent $c$ together with the eigenvector $\left(\alpha_{1}, \alpha_{2}, \alpha_{3}, \alpha_{4}\right)$ which, to avoid ambiguity, was normalized such that $\left(\sum_{i=1}^{4} \alpha_{i}^{2}\right)^{1 / 2}=1$. The eigenfunction $P(x)$ is then determined from $(20)$ and (19). A fourth order Runge-Kutta scheme was used to evaluate the functions $U_{i}$ and their derivatives needed in $(22)$.

In general the determinant of the coefficient matrix in (22) yields four roots for the Floquet multipliers $\mathrm{e}^{-2 c L}$ and thus four Floquet exponents $c$ with associated eigenfunctions $P(x)$. Figure 5 shows a plot of the periodic solution $u_{p}$ 


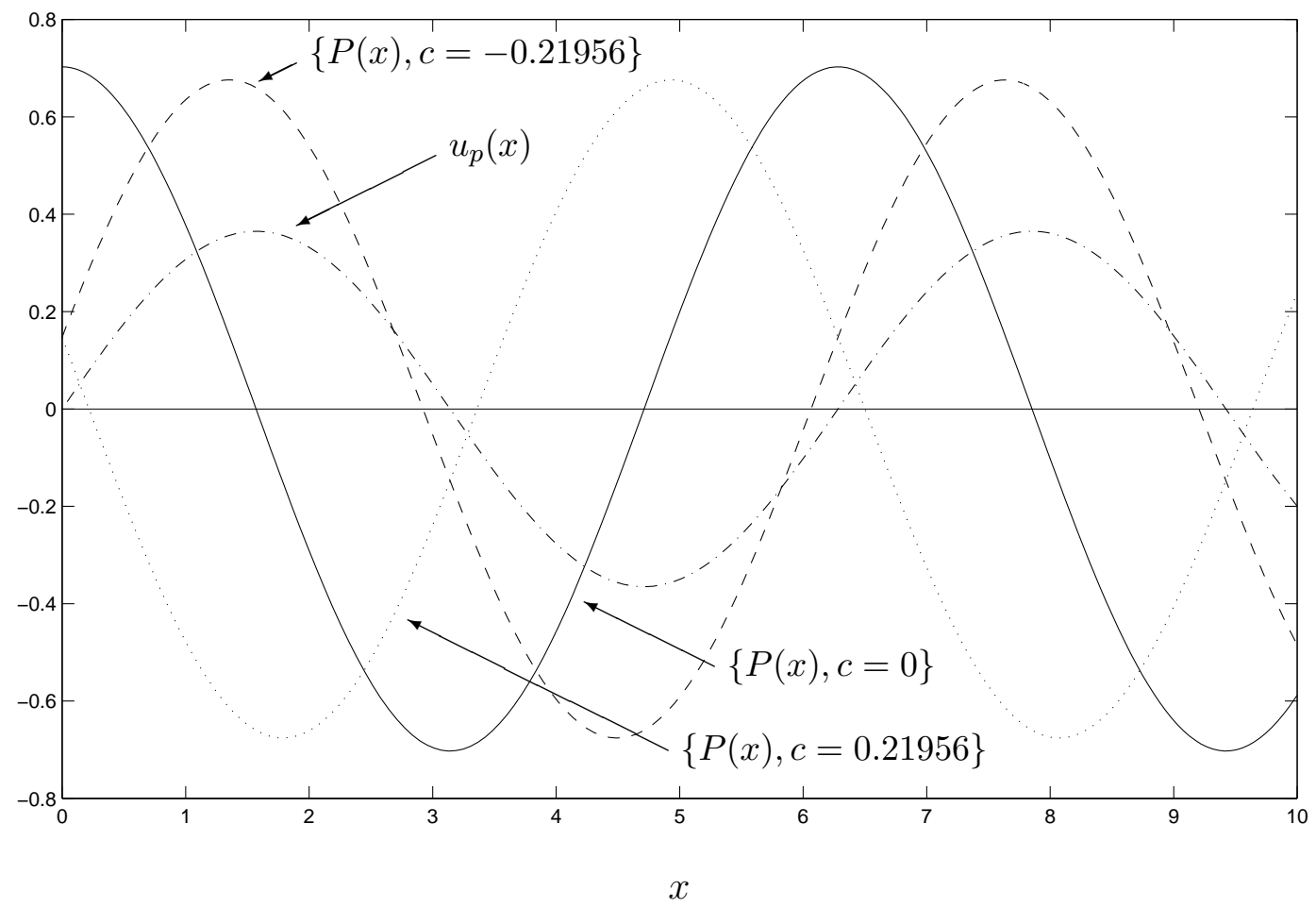

Fig. 5. Profiles of $u_{p}(x)$ and the Floquet eigenfunctions $P(x)$ at $L=\pi, \varepsilon=0.1$. The corresponding exponents $c$ are also indicated.

together with the eigenfunctions $P(x)$ at $\varepsilon=0.1, L=\pi$ which correspond to the exponents $c= \pm 0.21956$ and $c=0$. Note that $U=u_{p}^{\prime}$ is always a periodic solution of equation (18) and corresponds to the repeated root $c=0$, providing a useful check on the numerical algorithm. For general values of $\varepsilon$ and $L$, the other two Floquet exponents $c$ separate into regions where both are real (with equal and opposite values) and regions where both are purely imaginary, with equal and opposite values and $\left|\mathrm{e}^{-2 c L}\right|=1$. The boundary of these two regions where $c=0$ constitutes the Eckhaus stability boundary [18] since it is the locus of points at which spatially oscillatory disturbances to the periodic solution $u_{p}$ have zero temporal growth rate. This curve has also been found by Kramer and Hohenberg [7]. Within the Eckhaus boundary, where $c$ is real, the solution (19) with $c$ positive represents a spatially decaying solution which approaches the periodic form as $x \rightarrow \infty$. Figure 6 shows plots of the Floquet exponents for several values of $\varepsilon$ and Figure 7 shows the Eckhaus boundary 

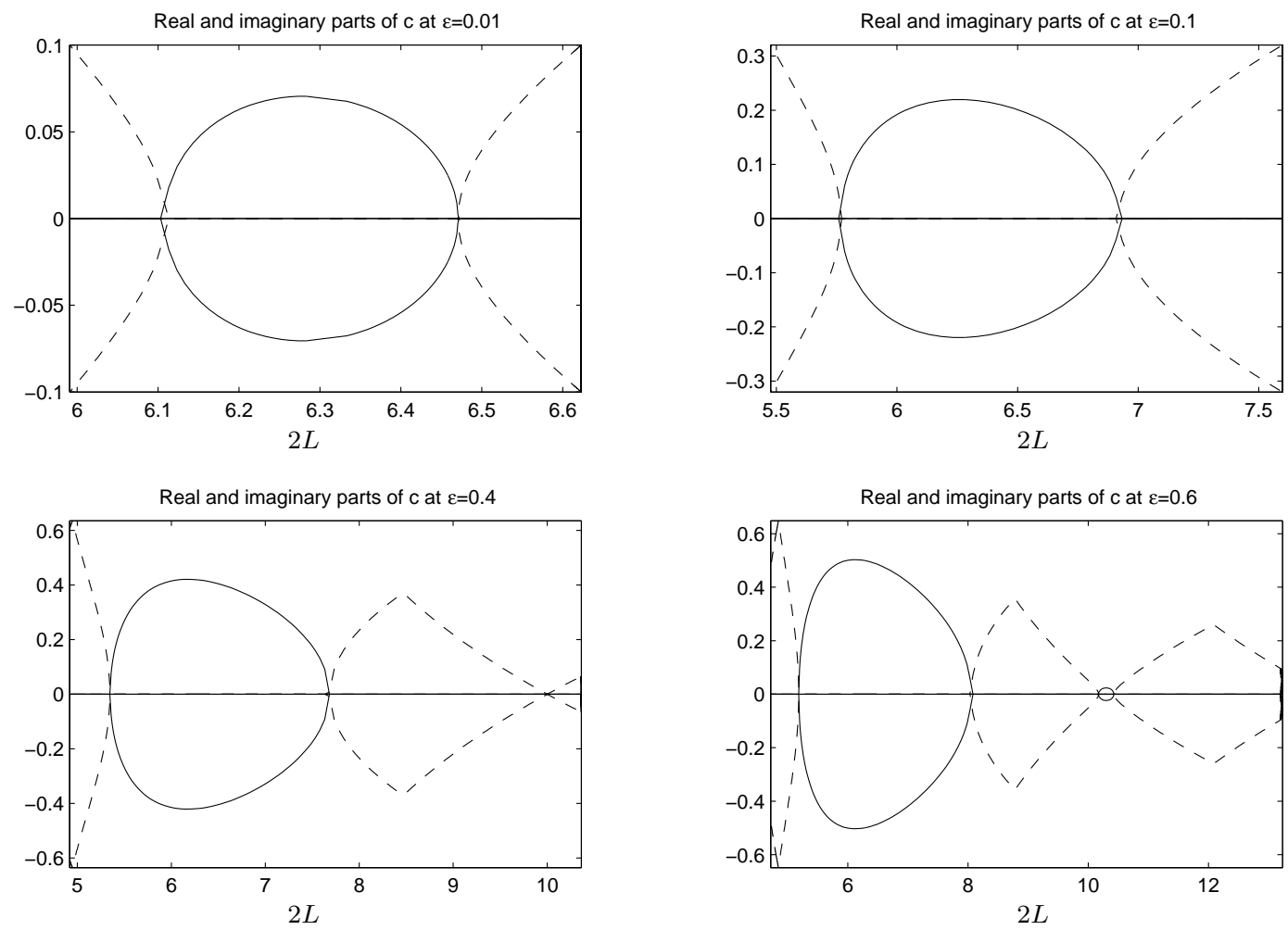

Fig. 6. Real (-) and imaginary (-- ) parts of the Floquet exponent $c$ as a function of $L$ for $\varepsilon=0.01,0.1,0.4$ and 0.6 .

$(c=0)$. Note that in Figure 6 for $\varepsilon=0.6$ there is a small region in which $c$ is real near $L=5.2$ which lies outside the main Eckhaus boundary. This is associated with the mode 1-2 interaction discussed in the previous section and suggests the possibility of further stable states at higher wavelengths; these are not investigated here.

In Figure 7 the Eckhaus boundary obtained from the Floquet analysis is compared with the result of a weakly nonlinear analysis. This is obtained by writing the solution of (12) as

$$
A_{0}=A_{p}(X)+\widetilde{K} B(X)+\ldots
$$

where $A_{p}$ is the periodic solution (13) and $\widetilde{K}$ is small and real. Substitution into (12) and neglect of the nonlinear terms in $\widetilde{K}$ then shows that the function 
$B(X)$ satisfies

$$
4 \frac{d^{2} B}{d X^{2}}+\left(1-6\left|A_{p}\right|^{2}\right) B-3 A_{p}^{2} B^{*}=0
$$

where the asterisk denotes the complex conjugate. Writing

$$
B=i \beta \mathrm{e}^{i Q X-C X}
$$

where $C$ is real and $\beta=\beta_{r}+i \beta_{i}$ is complex, equation (24) now gives

$$
\left(\begin{array}{ll}
\left(2 C^{2}+4 Q^{2}-1\right) & 4 Q C \\
-4 Q C & 2 C^{2}
\end{array}\right)\left(\begin{array}{l}
\beta_{r} \\
\beta_{i}
\end{array}\right)=\left(\begin{array}{l}
0 \\
0
\end{array}\right)
$$

and thus a non-trivial solution requires

$$
C^{2}\left(2 C^{2}+12 Q^{2}-1\right)=0
$$

Two roots correspond to $C=0$ (equivalent to $c=0$ in the finite $\varepsilon$ calculations) and the other two roots

$$
C= \pm \frac{1}{\sqrt{2}}\left(1-12 Q^{2}\right)^{1 / 2}
$$

are purely real if $Q^{2}<\frac{1}{12}$. If $Q^{2}>\frac{1}{12}$ these roots become purely imaginary although the analysis must then be generalized to allow for both $C$ and its conjugate in (25). The marginal case $C=0$ corresponds to the Eckhaus boundary, which is seen to be given by $Q= \pm 1 /(2 \sqrt{3})$ and (recalling that $Q$ is related to $L$ by (14)) is shown in Figure 7.

Within the Eckhaus boundary, the positive root (28) confirms the existence of a solution (23) which decays to the periodic form $A_{p}(X)$ as $X \rightarrow \infty$. Written in terms of $u$ using (10), this is equivalent to the solution (19) in which

$$
c \sim \varepsilon^{1 / 2} C=\frac{\varepsilon^{1 / 2}}{\sqrt{2}}\left(1-12 Q^{2}\right)^{1 / 2}
$$




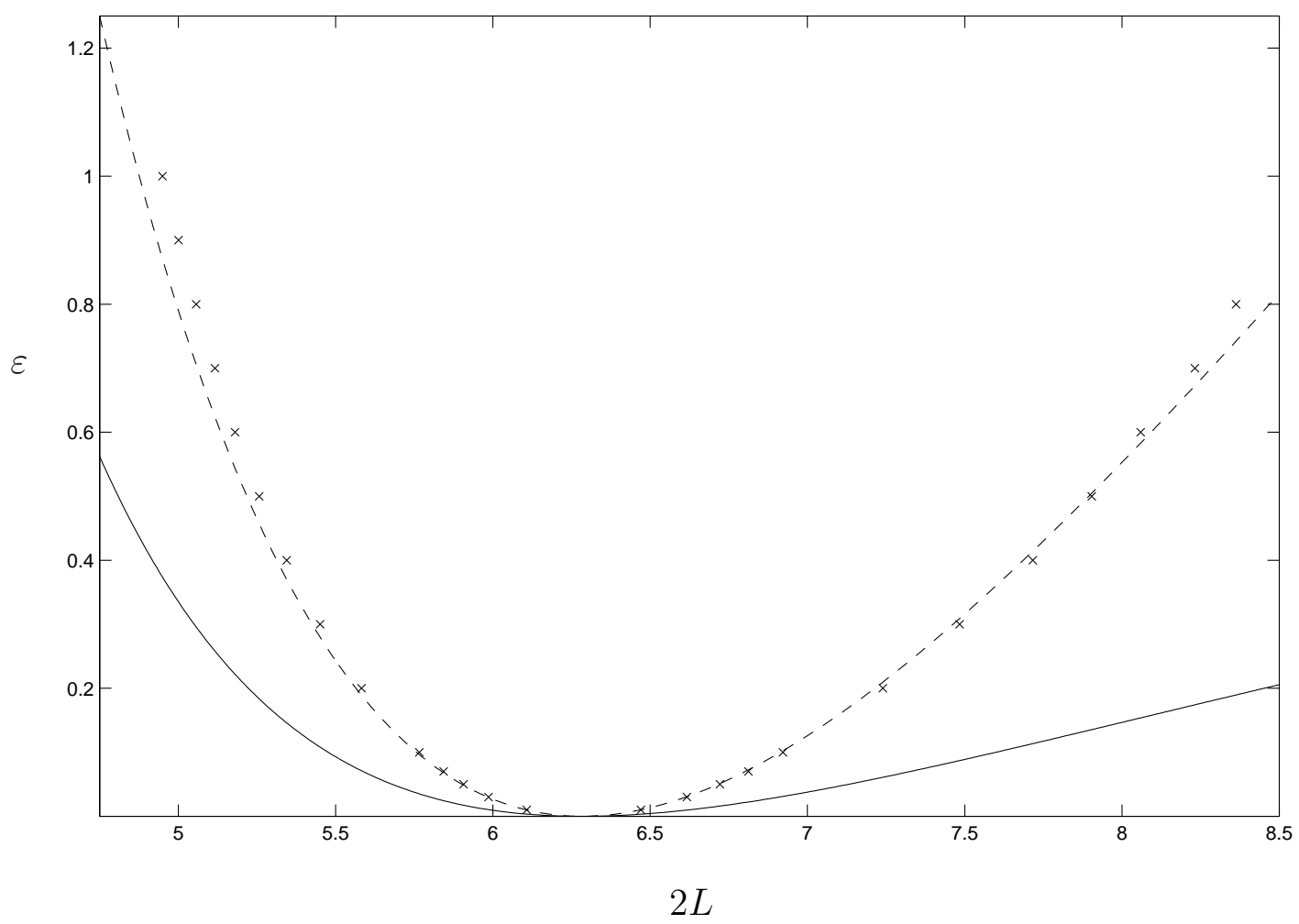

Fig. 7. Eckhaus boundary $(c=0)$ together with the weakly nonlinear approximation and the neutral curve. $(-)$ Neutral curve, $(\times \times)$ Eckhaus boundary, $(---)$ weakly nonlinear theory.

and, using (26),

$$
P(x) \sim \beta_{r} \varepsilon^{1 / 2}\left\{\sin \frac{n \pi x}{L}+\frac{2 \sqrt{2} Q}{\left(1-12 Q^{2}\right)^{1 / 2}} \cos \frac{n \pi x}{L}\right\}
$$

as $\varepsilon \rightarrow 0$. Note that from (26) the eigenfunction corresponding to the zero root $C=0$ is $P(x) \sim-\beta_{i} \varepsilon^{1 / 2} \cos \frac{n \pi x}{L}$, which is proportional to $u_{p}^{\prime}$.

\section{Nonlinear solutions at a lateral boundary}

Solutions of the one-dimensional Swift-Hohenberg equation

$$
\frac{d^{4} u}{d x^{4}}+2 \frac{d^{2} u}{d x^{2}}+(1-\varepsilon) u+u^{3}=0
$$


are now found in a semi-infinite domain $x \geq 0$ with boundary conditions

$$
u=\frac{d u}{d x}=0 \quad \text { at } x=0,
$$

and which approach the periodic form

$$
u \rightarrow u_{p}(x+\phi) \quad \text { as } x \rightarrow \infty
$$

Here $\phi$ is a phase shift of the periodic solution relative to the boundary and its determination is one of the key results of the analysis.

The periodic solution (33) must be attained through an exponential decay of the form

$$
u \sim u_{p}(x+\phi)+k \mathrm{e}^{-c x} P(x+\phi) \quad \text { as } x \rightarrow \infty
$$

where $u_{p}(x), c$ and $P(x)$ are known from the preceding analysis; the constants $k$ and $\phi$ must be chosen in order that the two conditions (32) are satisfied. In practice this was achieved by using a new variable $\bar{x}=x+\phi$ so that (34) becomes

$$
u \sim u_{p}(\bar{x})+\bar{k} \mathrm{e}^{-c \bar{x}} P(\bar{x})
$$

where $\bar{k}=k \mathrm{e}^{c \phi}$. The solution of (31) for $u(x)=\bar{u}(\bar{x})$ was then computed using a fourth order Runge-Kutta scheme for values of $\bar{x}$ decreasing from zero, using (35) and its derivatives to supply the initial conditions at $\bar{x}=0$. At the $m$ th stationary point of $\bar{u}\left(\bar{u}^{\prime}\left(\bar{x}_{m}\right)=0\right)$ the value of $\bar{u}$ is recorded. The calculation is repeated for a neighbouring value of $\bar{k}$ and a Newton iteration used to adjust the value of $\bar{k}$ until the second condition $\bar{u}=0$ is satisfied at $\bar{x}=\bar{x}_{m}$. Once the final converged values of $\bar{k}$ and $\bar{x}_{m}$ are known, the phase shift can be calculated from the relation $\phi=\bar{x}_{m}$ and then $k=\bar{k} \mathrm{e}^{-c \phi}$. In practice the value of $m$ must be chosen large enough (typically $m=10$ ) to ensure that $u$ is sufficiently close to the periodic form $u_{p}$ at $\bar{x}=0$; checks were made with different values of $m$ to test the validity of the results.

Plots of $k, \phi$ and $u(x)$ for $\varepsilon=0.5$ and $\varepsilon=1$ are shown in Figures 8 and 9. Dual solutions (labelled A and B) are found to exist for a restricted band 


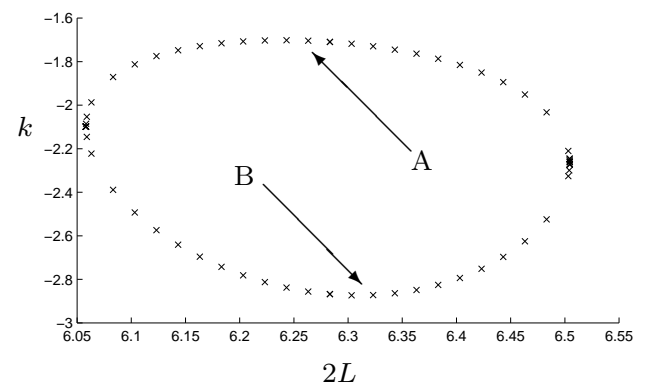

(c)

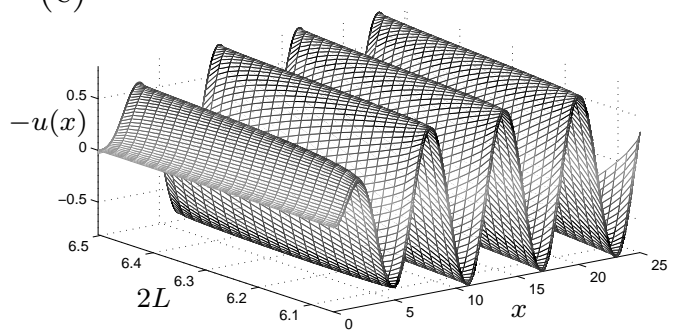

(e)

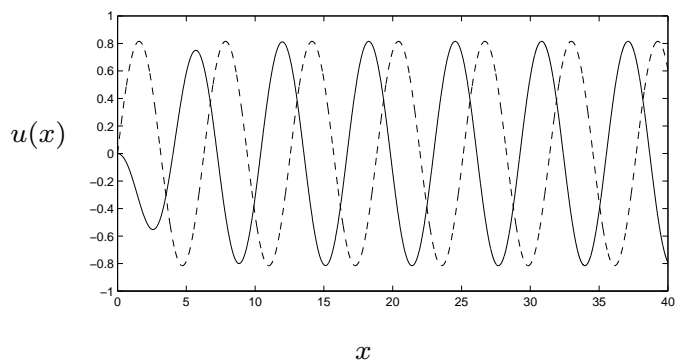

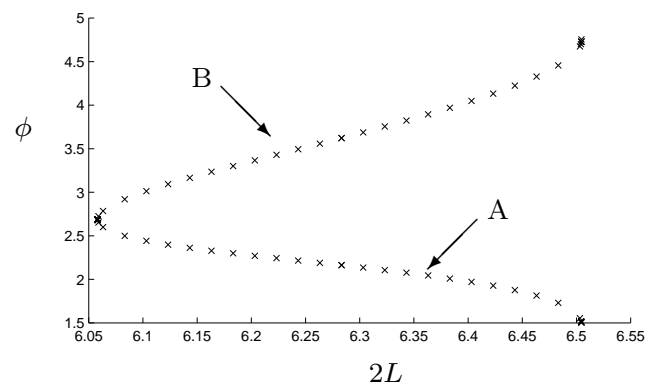

(d)

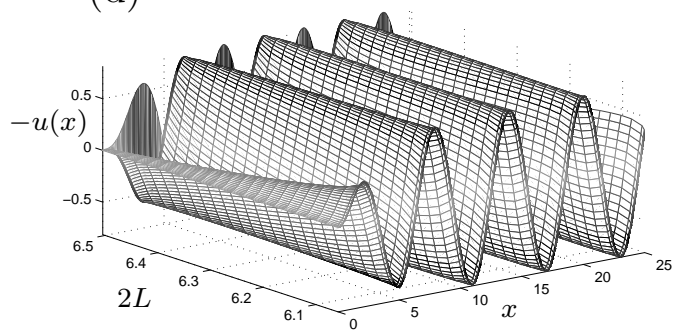

(f)

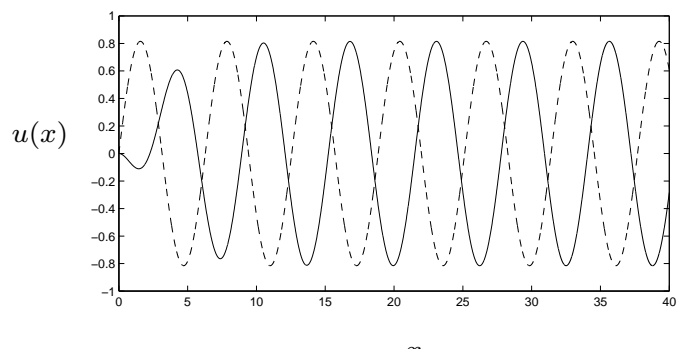

$x$

Fig. 8. Plots of $k, \phi$ and $u(x)$ for $\varepsilon=0.5$. (a) $k$ as a function of $2 L,(\mathrm{~b}) \phi$ as a function of $2 L,($ c) $u(x)$ as a function of $x$ and $2 L$ for branch $\mathrm{A},(\mathrm{d}) u(x)$ as a function of $x$ and $2 L$ for branch $\mathrm{B}$, (e) $u(x)$ on branch $\mathrm{A}$ as a function of $x$ for $L=\pi$, (f) $u(x)$ on branch B as a function of $x$ for $L=\pi$. From (e) and (f) it is seen that the solution adjusts to the phase-shifted periodic solution within a couple of wavelengths of the wall; the periodic solution $u_{p}(x)$ is shown by a dashed curve for comparison.

of wavelengths $2 L$ within the Eckhaus boundary. The width of the restricted band of wavelengths increases as $\varepsilon$ increases and its form in the weakly nonlinear limit $(\varepsilon \rightarrow 0)$ was first determined by Cross et al [6]. Here the main results of the weakly nonlinear analysis are summarized in order to make a detailed comparison with the numerical results.

In the region where $X=\varepsilon^{1 / 2} x=O(1)$, the solution for $u$ develops in the form (6) and in the steady-state case

$$
u_{0,1}=A_{0,1}(X) \mathrm{e}^{i x}+c . c
$$




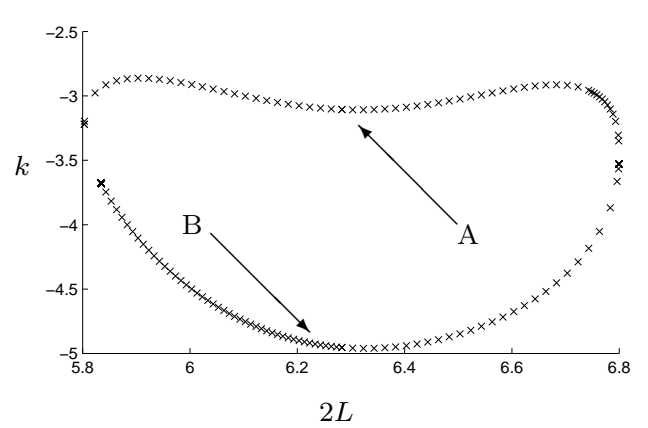

(c)

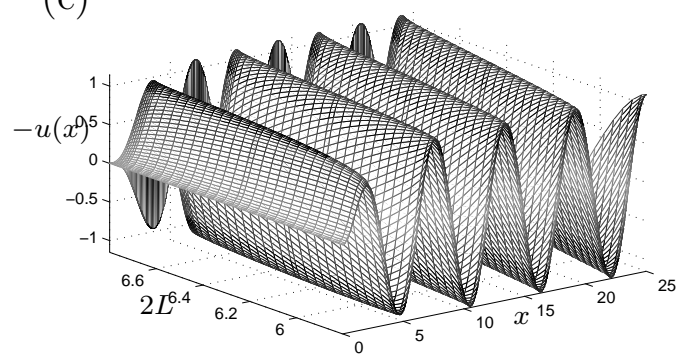

(e)

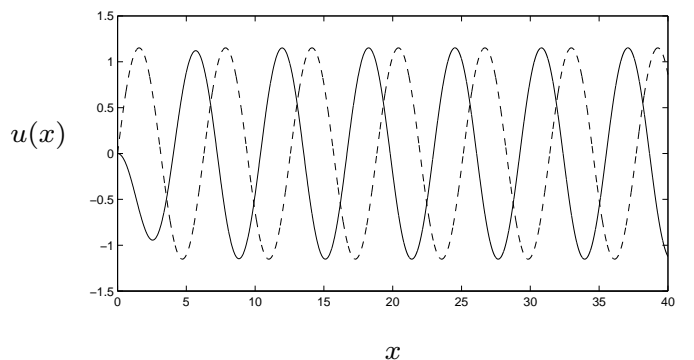

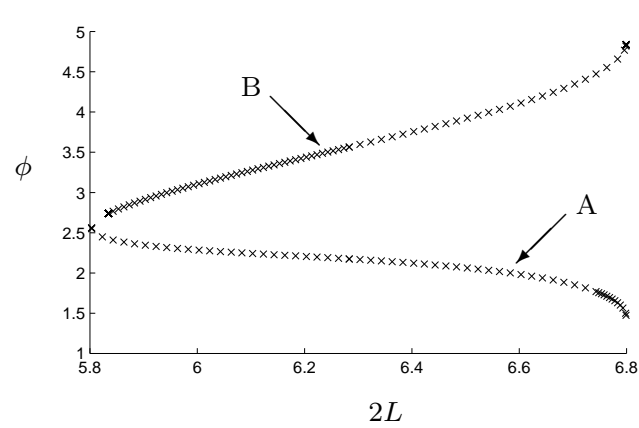

(d)

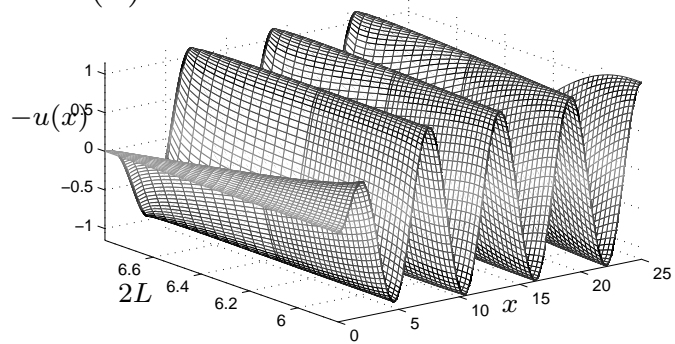

(f)

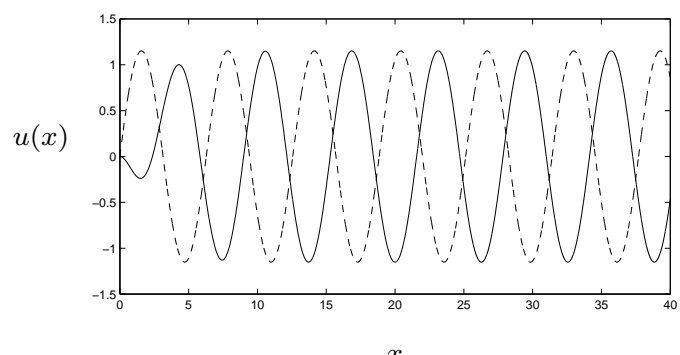

Fig. 9. Plots of $k, \phi$ and $u(x)$ for $\varepsilon=1$. (a) $k$ as a function of $2 L$, (b) $\phi$ as a function of $2 L$, (c) $u(x)$ as a function of $x$ and $2 L$ for branch $\mathrm{A},(\mathrm{d}) u(x)$ as a function of $x$ and $2 L$ for branch $\mathrm{B},(\mathrm{e}) u(x)$ on branch $\mathrm{A}$ as a function of $x$ for $L=\pi$, (f) $u(x)$ on branch $\mathrm{B}$ as a function of $x$ for $L=\pi$. The periodic solution $u_{p}(x)$ is shown by a dashed curve in (e) and (f).

where $A_{0}$ and $A_{1}$ satisfy the equations

$$
4 \frac{d^{2} A_{0}}{d X^{2}}+A_{0}-3 A_{0}\left|A_{0}\right|^{2}=0
$$

and

$$
4 \frac{d^{2} A_{1}}{d X^{2}}+A_{1}-6\left|A_{0}\right|^{2} A_{1}-3 A_{0}^{2} A_{1}^{*}=4 i \frac{d^{3} A_{0}}{d X^{3}}
$$

Equation (37) is just the steady-state version of (12), and equation (38) comes from the solvability condition for $u_{3}$ at order $\varepsilon^{2}$. The relevant solution for $A_{0}$ 


$$
A_{0}=\frac{1}{\sqrt{3}} \mathrm{e}^{i \theta} \tanh \frac{X}{2 \sqrt{2}}
$$

where $\theta$ is an arbitrary phase constant and then

$$
\begin{aligned}
A_{1}= & \mathrm{e}^{i \theta}\left\{k_{r} \operatorname{sech}^{2} \frac{X}{2 \sqrt{2}}+i\left[\frac{1}{4 \sqrt{6}}-\frac{3}{4 \sqrt{6}} \tanh ^{2} \frac{X}{2 \sqrt{2}}+k_{i} \tanh \frac{X}{2 \sqrt{2}}\right.\right. \\
& \left.\left.+l\left(X \tanh \frac{X}{2 \sqrt{2}}-2 \sqrt{2}\right)\right]\right\}
\end{aligned}
$$

where $k_{r}, k_{i}$ and $l$ are further real constants. As $X \rightarrow \infty$ the solution (6) develops into a periodic solution of uniform amplitude

$$
u \sim \frac{1}{\sqrt{3}} \varepsilon^{1 / 2} \mathrm{e}^{i q x+i \theta}+c . c .
$$

For an infinite layer it was shown in Section 2 that solutions of more general amplitude $\varepsilon^{1 / 2}\left(1-4 Q^{2}\right)^{1 / 2} / \sqrt{3}$ exist for $|Q|<\frac{1}{2}$ with $q=1+\varepsilon^{1 / 2} Q$ but the relevant solutions here correspond to small values of $Q$, of order $\varepsilon^{1 / 2}$. Indeed, since from (39) and (40) as $X \rightarrow \infty$,

$$
\begin{aligned}
u & \sim \frac{1}{\sqrt{3}} \varepsilon^{1 / 2} \mathrm{e}^{i x+i \theta}\left(1+i \varepsilon^{1 / 2} \sqrt{3} l X\right)+\text { c.c. } \\
& \sim \frac{1}{\sqrt{3}} \varepsilon^{1 / 2} \mathrm{e}^{i x+i \theta}(1+i \varepsilon \sqrt{3} l x)+c . c .
\end{aligned}
$$

it is seen that matching with (41) requires that, correct to order $\varepsilon$, the wavenumber $q$ must be given by

$$
q=1+\varepsilon \sqrt{3} l
$$

A formal derivation of the solution (41) and (43) obtained by assuming a twoscale expansion with length scales $x \sim 1$ and $x \sim \varepsilon^{-1}$ is given in Appendix E of Cross et al [6] although an alternative procedure [19] is to include the correction (43) to the wavenumber directly in the expansion (6) (and an inner expansion (44) below) in which case an expansion can be formulated which remains uniformly valid as $X \rightarrow \infty$. 
The solution (6) breaks down on the scale $x=O(1)$ near the boundary where locally the solution has the form

$$
u=\varepsilon \bar{u}_{0}(x)+\ldots \quad \text { as } \varepsilon \rightarrow 0
$$

and $\bar{u}_{0}$ satisfies the linearized version of (31) in which the term $\varepsilon u$ can also be ignored. Thus

$$
\bar{u}_{0}=(a+b x) \mathrm{e}^{i x}+\text { c.c. },
$$

where $a$ and $b$ are complex constants. Matching with the outer form (6) and making use of (39), (40) gives

$$
b=\frac{1}{2 \sqrt{6}} \mathrm{e}^{i \theta}, \quad a=\left(k_{r}-i\left\{2 \sqrt{2} l-\frac{1}{4 \sqrt{6}}\right\}\right) \mathrm{e}^{i \theta}
$$

whilst application of the boundary conditions (32) requires

$$
a+a^{*}=0, \quad i\left(a-a^{*}\right)+b+b^{*}=0 .
$$

Writing $a=a_{r}+i a_{i}$ and $b=b_{r}+i b_{i}$, it follows that

$$
\begin{aligned}
& a_{r}=0=k_{r} \cos \theta-\left(\frac{1}{4 \sqrt{6}}-2 \sqrt{2} l\right) \sin \theta \\
& a_{i}=\frac{1}{2 \sqrt{6}} \cos \theta=k_{r} \sin \theta+\left(\frac{1}{4 \sqrt{6}}-2 \sqrt{2} l\right) \cos \theta
\end{aligned}
$$

and thus

$$
l=-\frac{1}{16 \sqrt{3}} \cos 2 \theta, \quad k_{r}=\frac{1}{4 \sqrt{6}} \sin 2 \theta .
$$

This gives a range of solutions with $|l| \leq 1 /(16 \sqrt{3})$, that is, the wavenumber restriction

$$
1-\frac{1}{16} \varepsilon \leq q \leq 1+\frac{1}{16} \varepsilon
$$

in the limit as $\varepsilon \rightarrow 0$. For a given wavenumber and its corresponding value of $l$ there are two possible values of $\theta$, say $\theta=\theta_{0}$ and $\theta=\pi-\theta_{0}$, (with 

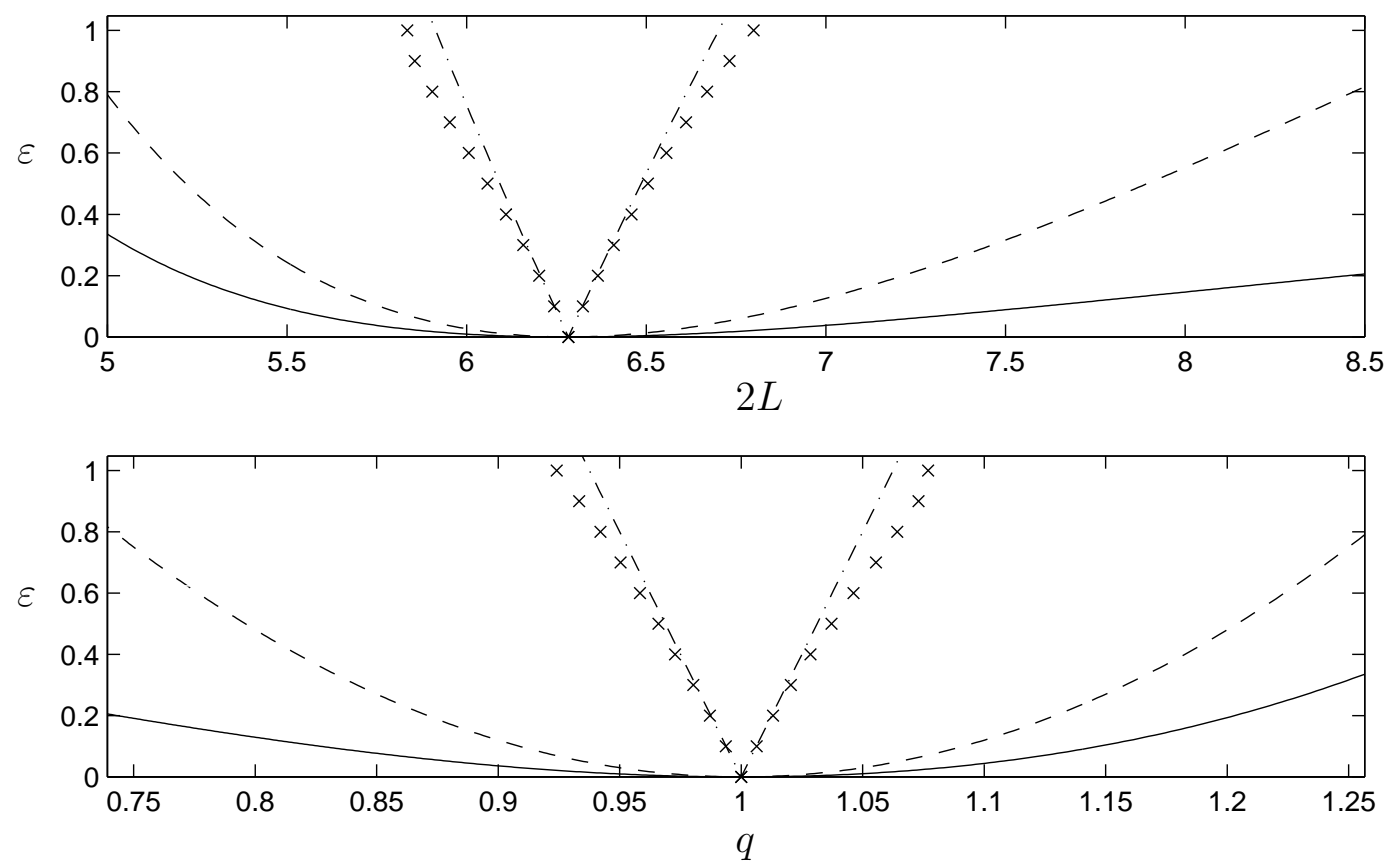

Fig. 10. Region of existence of solutions in the presence of a lateral boundary. Also shown is the weakly nonlinear approximation, together with the Eckhaus boundary and the neutral curve. (- Neutral curve, (-- ) Eckhaus boundary, $(-\cdot-\cdot)$ weakly nonlinear theory, $(\times \times)$ present theory.

$\left.0 \leq \theta_{0} \leq \frac{\pi}{2}\right)$ corresponding to $k_{r}= \pm \frac{1}{4 \sqrt{6}} \sin 2 \theta_{0}$, respectively. These two solutions coalesce when $\theta_{0}$ reaches $\frac{\pi}{2}$ and $l=1 /(16 \sqrt{3})$, and when $\theta_{0}$ reaches zero and $l=-1 /(16 \sqrt{3})$ at the other extreme; in both limits $k_{r}$ approaches zero. Figure 10 shows the weakly nonlinear result (51) along with the results of the numerical calculations for finite $\varepsilon$.

More detailed comparisons with the weakly nonlinear theory are shown in Figure 11, where the asymptotic forms of $k, u^{\prime \prime}(0)$ and $u^{\prime \prime \prime}(0)$ as $\varepsilon \rightarrow 0$ are compared with the numerical calculations for $\varepsilon=0.1$. From (44)-(50),

$$
u^{\prime \prime}(0) \sim-4 \varepsilon b_{i}=-\frac{2}{\sqrt{6}} \varepsilon \sin \theta, \quad \varepsilon \rightarrow 0
$$



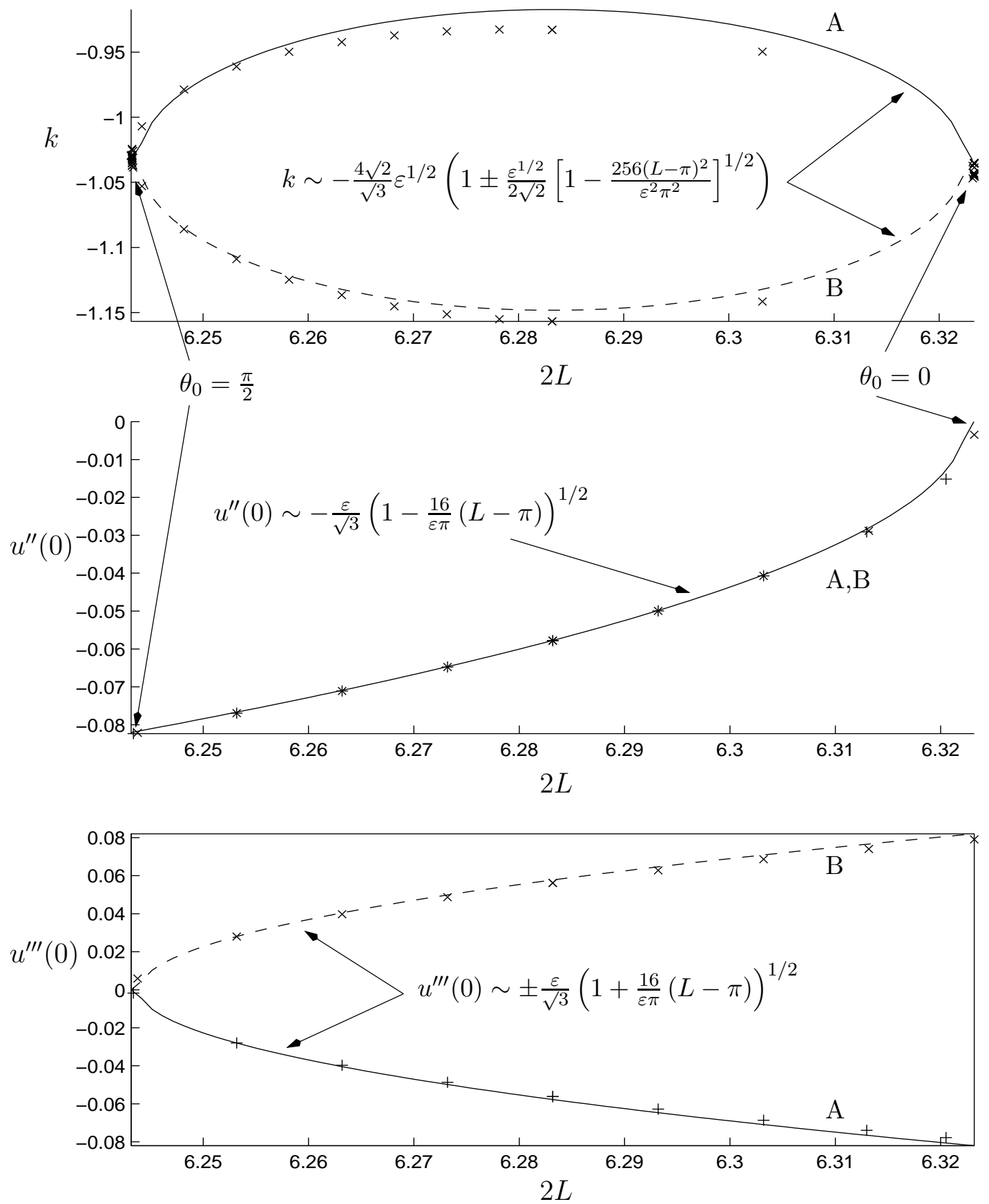

Fig. 11. Comparison of $k, u^{\prime \prime}(0)$ and $u^{\prime \prime \prime}(0)$ with weakly nonlinear theory for $\varepsilon=0.1$. 
and

$$
u^{\prime \prime \prime}(0) \sim-4 \varepsilon b_{r}=-\frac{2}{\sqrt{6}} \varepsilon \cos \theta, \quad \varepsilon \rightarrow 0 .
$$

Taking into account the decay of the tanh functions in (39), (40) as $X \rightarrow \infty$ and writing the outer solution (6) in terms of the variable $x$ gives

$$
\begin{gathered}
u \sim \frac{2 \varepsilon^{1 / 2}}{\sqrt{3}} \sin \left\{(1+\varepsilon \sqrt{3} l) x+\theta+\frac{\pi}{2}+O\left(\varepsilon^{1 / 2}\right)\right\} \times \\
\left(1-2\left[1-\frac{\varepsilon^{1 / 2}}{2 \sqrt{2}} \sin 2 \theta\right] \mathrm{e}^{-\frac{\varepsilon^{1 / 2} x}{\sqrt{2}}}\right)
\end{gathered}
$$

as $x \rightarrow \infty$. The Floquet exponent and eigenfunction given by (29) and (30) (evaluated at $Q=0$ and with $P$ normalized in the same manner as in the numerical calculations) are

$$
c \sim \frac{\varepsilon^{1 / 2}}{\sqrt{2}}, \quad P(x) \sim\left(\frac{3}{8 \varepsilon}\right)^{1 / 2} u_{p}(x), \quad \varepsilon \rightarrow 0
$$

and comparison with (54) shows that the phase shift $\phi$ is given by

$$
\phi \sim \theta+\frac{\pi}{2}, \quad \varepsilon \rightarrow 0
$$

and the coefficient $k$ is given by

$$
k \sim-\frac{4 \sqrt{2}}{\sqrt{3}} \varepsilon^{1 / 2}\left(1-\frac{\varepsilon^{1 / 2}}{2 \sqrt{2}} \sin 2 \theta\right), \quad \varepsilon \rightarrow 0 .
$$

For the two solution branches $\mathrm{A}\left(\theta=\theta_{0}\right)$ and $\mathrm{B}\left(\theta=\pi-\theta_{0}\right)$, the two values of $k$ coalesce at $-4 \sqrt{2} \varepsilon^{1 / 2} / \sqrt{3}$ when $\theta_{0}=0 \quad(l=-1 /(16 \sqrt{3}))$ and when $\theta_{0}=\frac{\pi}{2} \quad(l=1 /(16 \sqrt{3}))$. The phase shifts of the two solutions are $\phi=\theta_{0}+\frac{\pi}{2}$ and $\phi=\frac{3 \pi}{2}-\theta_{0}$ so that these differ by $\pi$ at $\theta_{0}=0$ and are equal at $\theta_{0}=\frac{\pi}{2}$. Solutions for $u$ with opposite sign are obtained by changing the range of $\theta$ from $[0, \pi]$ to $[\pi, 2 \pi]$. For the purpose of the comparison of (52), (53) and (57) with the numerical results in Figure 11, the formulae given in (43) and (50) have been used to relate the phase constant $\theta$, via $l$, to the wavenumber $q$. Figure 11 indicates excellent agreement between the numerical results at $\varepsilon=0.1$ and the results of the weakly nonlinear theory. 
The stability of the dual solutions described in this section can be analyzed in the weakly nonlinear limit using the approach described by Daniels [20] for the stress-free Rayleigh-Bénard problem. A general disturbance $\widetilde{u}(x, t)$ to the steady-state solution $u(x)$ is introduced and with the temporal derivative $\partial u / \partial t$ added to the left-hand side of (31) the disturbance is expanded in powers of $\varepsilon$ within the inner and outer regions. It is readily shown that disturbances in phase with the steady-state solution decay on a time-scale $t \sim \varepsilon^{-1}$ whereas 'out-of-phase' disturbances are neutral on this time scale and must be examined on the longer time-scale $t \sim \varepsilon^{-2}$. This leads to the possibility of spatially decaying, temporally growing disturbances that have the form

$$
\begin{aligned}
\widetilde{u} \sim & i \exp \left\{i(1+\varepsilon \sqrt{3} l) x+i \theta+\left(\frac{1}{8} \varepsilon \sin 2 \theta\right) x\right. \\
& \left.+\left(\frac{1}{16} \varepsilon^{2} \sin ^{2} 2 \theta\right) t\right\}+c . c .
\end{aligned}
$$

on the scale $x \sim \varepsilon^{-1}$, provided that

$$
\sin 2 \theta<0
$$

For $\sin 2 \theta>0$, disturbances that remain bounded spatially are found to decay temporally. Thus within the one-dimensional framework studied here, of the dual solutions $\theta=\theta_{0}$ and $\theta=\pi-\theta_{0}$ (with $0 \leq \theta_{0} \leq \frac{\pi}{2}$ ) only the former (corresponding to the upper branch of values of $k$ in Figure 11) is stable. Physically, this corresponds to the branch of solutions for which a flow reversal very close to the lateral boundary is avoided.

\section{Summary}

A method has been developed for finding steady-state solutions of the onedimensional Swift-Hohenberg equation which satisfy no-slip conditions at a lateral boundary and approach a periodic form at large distances from the boundary. Use of Floquet theory enables the asymptotic behaviour of the solution to be analyzed and the existence of dual nonlinear solutions to be identified for given wavelengths of the periodic form. The lateral boundary severely restricts the waveband of solutions compared with that which exists 
for an infinite layer. A key result of the analysis is the determination of the phase shift of the periodic form relative to the boundary, so that at large distances from the boundary both the amplitude and phase of the possible periodic forms are known as a function of wavelength.

At finite values of the control parameter $\varepsilon$, the adjustment to the periodic form is completed within just a few roll-widths of the lateral boundary, the scale of decay being determined by the Floquet exponent $c$. This scale increases as $\varepsilon$ decreases, with $x \sim \sqrt{2} \varepsilon^{-1 / 2}$ as $\varepsilon \rightarrow 0$. A detailed comparison with asymptotic results has been carried out in this limit, showing that weakly nonlinear theory remains a reasonable approximation up to $\varepsilon=1$; however, further work is needed to investigate the possible significance of mode interactions in the Swift-Hohenberg system for $\varepsilon>\frac{9}{25}$.

The present work shows that, in principle, extended roll patterns in large-scale systems can be determined in the nonlinear regime by analyzing the structure of the solution close to the lateral boundaries. This is an important observation in the context of real pattern-forming systems, such as the Rayleigh-Bénard system, where the heavy demands of three-dimensional computations still preclude the accurate numerical determination of patterns involving more than just a few convective rolls. Typical calculations scale with the size of the domain, whereas the present method requires the calculation of the solution for the equivalent of only a few roll widths.

In order to make a direct comparison with numerical computations on a large but finite domain it is necessary to determine the relevant quantized wavelength $2 L$ to be used in the present theory. In general this can be calculated by requiring that the phase shift of the periodic solution induced by each boundary is the same (modulo $2 L$ ), leading to a consistent solution across the entire domain. Taking the domain to be $0 \leq x \leq d$ with $d \gg L$, the phase shift of $u_{p}$ due to the boundary at $x=0$ is $\phi$, the function introduced in (33) and displayed, for example, in figures 8 and 9. Similarly, the second boundary, at $x=d$ will induce the behaviour

$$
u \sim u_{p}(d-x+\phi)=-u_{p}(x-d-\phi)=u_{p}(x-d-\phi+L) \quad \text { as } \quad d-x \rightarrow \infty,(60)
$$

equivalent to a phase shift of $L-d-\phi$ in the $x$ direction. Allowing for the 
possibility of a sign reversal of $u$ at either end, the phase shift between the two ends must differ by an integer multiple of $L$, and so a consistent overall solution requires

$$
\phi=n L+L-d-\phi
$$

where $n$ is an integer. Each function $\phi$ involved here may take the value on either of its two branches ( $\phi_{A}$ or $\phi_{B}$, say, corresponding to branches A and B in figures 8 and 9 ). This leads to an (unstable) class of non-symmetric solutions (where $\phi_{A}$ is used on one side of (61) and $\phi_{B}$ on the other), an unstable class of symmetric solutions (where $\phi_{B}$ is used on both sides) and a stable class of symmetric solutions (either odd or even in $x$ ) where $\phi_{A}$ is used on both sides. There have been discussed in the weakly nonlinear limit in [6] and [20].

It should be noted that for given $\varepsilon$ and a given domain length $d$, (61) is an implicit equation for $L$ (and $n$ ). However, to test the present theory against computations on a finite domain, $d$ can be chosen so that a turning point of the semi-infinite solution on $x \geq 0$ coincides with the mid-point of the domain. In figure $12, d$ has been chosen as 61.6473 so that this occurs for the semi-infinite solution with $\varepsilon=0.5$ and $L=\pi$. It is seen that the semi-infinite solution is in excellent agreement with a more time-consuming computation carried out on the finite domain using a combination of time-stepping a finite-difference representation of the Swift-Hohenberg equation and Newton iteration. The expected discrepancy very close to the boundary at $x=d$ can easily be removed by using the semi-infinite solution locally. The solution in figure 12 corresponds to taking $n=20$ and $\phi=\phi_{A}=2.1631$ in (61), this being the relevant value of $\phi$ at $L=\pi$ in figure 8(b). The value of $n$ in (61) is a measure of the number of zeros of $u(x)$ in the interval $[0, d]$.

A long-term aim is to extend the present method to the fully nonlinear RayleighBénard problem. Progress has already been made by the present authors in extending the analysis described here for the Swift-Hohenberg equation to two dimensions $(x, y)$ where $(1)$ applies and the flow domain consists of a semiinfinite channel $x \geq 0, \quad 0 \leq y \leq L_{y}$. This will be the subject of a future paper. 


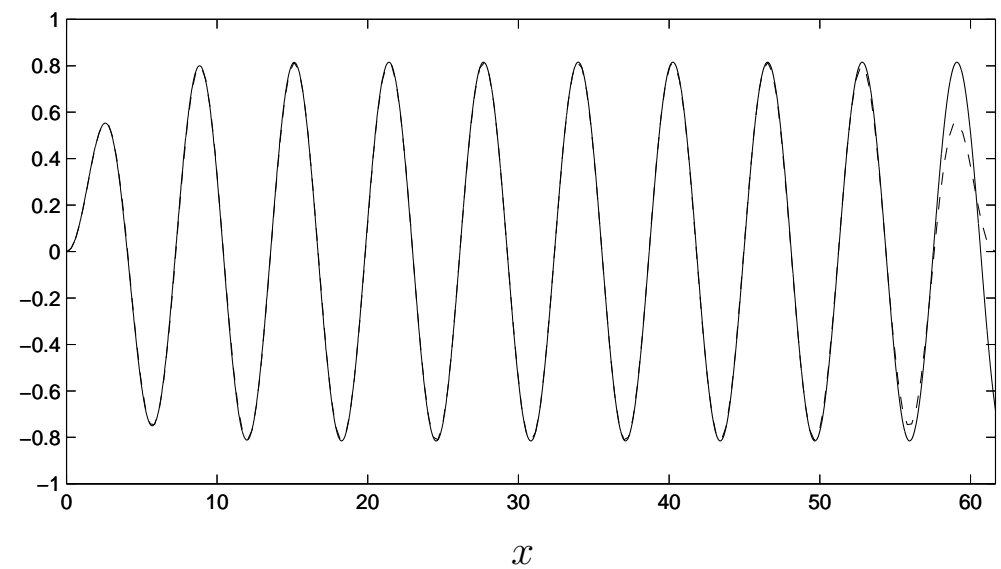

Fig. 12. Comparison of the semi-infinite solution $(-)$ for $\varepsilon=0.5, L=\pi$ with a steady solution calculated on a finite domain of length $61.6473(--)$.

\section{Acknowledgements}

This work was supported by a research grant from the EPSRC Applied Nonlinear Mathematics Programme.

\section{References}

[1] M.C. Cross and P.C. Hohenberg, Pattern formation outside of equilibrium, Rev. Mod. Phys. 65 (1993) 851-1112.

[2] E.L. Koschmieder, Bénard Cells and Taylor Vortices (Cambridge University Press, 1993).

[3] W. Arter and A.C. Newell, Numerical simulation of Rayleigh-Bénard convection in shallow tanks, Phys. Fluids 31 (1988) 2474-2485.

[4] J. Swift and P.C. Hohenberg, Hydrodynamic fluctuation at the convective instability, Phys. Rev. A 15 (1977) 319-328.

[5] M.C. Cross, P.G. Daniels, P.C. Hohenberg and E.D. Siggia, Effect of distant sidewalls on wavenumber selection in Rayleigh-Bénard convection, Phys.Rev. Lett 45 (1980) 898-902.

[6] M.C. Cross, P.G. Daniels, P.C. Hohenberg and E.D. Siggia, Phase-winding solutions in a finite container above the convective threshold, J. Fluid Mech. 127 (1983) 155-183.

[7] L. Kramer and P.C. Hohenberg, Effects of boundary conditions on spatially periodic states, Physica D 13 (1984) 357-369. 
[8] K. Tsiveriotis and R.A. Brown, Bifurcation structure and the Eckhaus instability, Phys. Rev. Lett. 63 (1989) 2048-2051.

[9] E. Hernandez-Garcia, M. San Miguel, R. Toral and J. Vinals, Noise and pattern selection in the one-dimensional Swift-Hohenberg equation, Physica D 61 (1992) 159-165.

[10] H.S. Greenside, W.M. Coughran and N.L. Schryer, Nonlinear pattern formation near onset of Rayleigh-Bénard convection, Phys. Rev. Lett. 49 (1982) 726-729.

[11] H.S. Greenside and W.M. Coughran, Nonlinear pattern formation near the onset of Rayleigh-Bénard convection, Phys. Rev. A 30 (1984) 398-428.

[12] M. Kuwamura, The stability of roll solutions of the two-dimensional SwiftHohenberg equation and the phase diffusion equation, SIAM J. Math. Anal. 27 (1996) 1311-1335.

[13] G. Dangelmayr, Steady-state mode interactions in the presence of $O(2)$ symmetry, Dyn. Stab. Syst. 1 (1986) 159-185.

[14] S.M. Cox, Mode interactions in Rayleigh-Bénard convection, Physica D 95 (1996) 50-61.

[15] L.F. Shampine and M.W. Reichelt, The Matlab ODE Suite, http://www.mathworks.com/odesuite.html, (The Math Works Inc., 1996)

[16] D. Ho, On the influence of lateral boundaries in nonlinear convection, $\mathrm{PhD}$ thesis, City University, London (2000).

[17] E.L. Ince, Ordinary Differential Equations (Dover Publications, 1956)

[18] V. Eckhaus, 1965 Studies in Nonlinear Stability Theory, Springer Tracts in Natural Philosophy 6 (Springer, 1965).

[19] M.R.E. Proctor, Private communication (2000).

[20] P.G. Daniels, The effect of distant side-walls on the evolution and stability of finite-amplitude Rayleigh-Bénard convection, Proc. Roy. Soc. Lond. A378 (1981) 539-566. 